\section{Neonatal Seizures: Continued debate on Phenobarbitone versus Phenytoin}

Authors of a recent paper [1] concluded that phenobarbitone is more efficacious than phenytoin in control of clinical seizures in term or near-term neonates. However, it has been inferred from apparent misinterpretation of data. The preponderance of seizure type was subtle seizure, which should not be considered as clinical seizure equivalent. Authors themselves mentioned of observations by Mizrahi, et al. [2] that near $85 \%$ of subtle seizures may not have electrophysiological correlate and may represent brainstem release phenomenon. Authors also reported that $91.6 \%$ neonates had normal interictal EEG. Phenobarbitone due to its GABA mimetic property may be effective in these events. So, for a pragmatic study design with lack of video EEG facility, at least subtle seizures should be excluded from study. Additionally, authors have defined cessation of clinical seizure activity as primary outcome variable, but lack of defining timeline threatens its internal validity. Also, authors should have reported seizure recurrences in each intervention arm.

JitenDRA KUMAR SAHU
Assistant Professor,
Pediatric Neurology Division,
Department of Pediatrics,
PGIMER, Chandigarh 160012, India.
jsh2003@gmail.com

\section{REFERENCES}

1. Pathak G, Upadhyay A, Pathak U, Chawla D, Goel SP. Phenobarbitone versus phenytoin for treatment of neonatal seizures: An open-label randomized controlled trial. Indian Pediatr. 2013;50:753-57.

2. Mizrahi EM, Kellaway P. Characterization and classification of neonatal seizures. Neurology. 1987;37: $1837-44$.

\section{REPLY}

Though, Mizrahi, et al. [1] documented that most of term newborn infants with subtle seizures had inconsistent association with EEG seizure activity, it was a small study of only 22 neonates. We would like to draw your attention to a larger study by Scher, et al. [2] on 44 neonates with subtle clinical seizures (also called as motor automations), who reported that subtle seizures accounted for $70-75 \%$ of all clinical seizures that had simultaneous EEG correlates. So, it will be too simplistic to conclude that "all the subtle seizure should not be considered as clinical seizure equivalent". At best, it can be stated that more research is required in this field.

A relevant question is whether absence of electrical seizure activity rules out an epileptic origin for the clinical activity? The answer to this is not known unequivocally as well! The epileptic phenomenon generated at subcortical level (e.g. inferior colliculus as in case of subtle seizure) may not be recorded in the absence of surface electrode recorded EEG discharges. Studies have reported that the proportion of infants who exhibited subtle clinical seizures is nearly identical among infants who either did or did not exhibit simultaneous electrographic discharge [2,3]. Thus, we do not think in our study there was over-representation of the subtle clinical seizures in the infants who did not exhibit an EEG correlate. Moreover the groups with and without electrographic accompaniments of the subtle seizures were clinically similar and had similar neurological outcome. There was therefore, no indication that the infants who did not have consistent EEG correlates were more likely to have cerebral destruction and thereby "release phenomenon" than were the infants who had consistent EEG correlates. No National or International guidelines recommend that subtle seizures should not be treated. We do not think that we need to exclude babies with subtle seizures from our study.

We had defined cessation of clinical seizure activity as primary outcome variable of this study. Seizures were considered to be controlled if the baby was seizures free $24 \mathrm{~h}$ after last seizures.

Seizure recurrence is part of composite outcomes at 3 and 6 months, as mentioned in the "methods" section, but it has not been reported separately. It was not possible now to tease out that data separately.

AMIT UPADHYAY anuamit7@rediffmail.com

\section{REFERENCES}

1. Mizrahi EM, Kellaway P. Characterization and classification of neonatal seizures. Neurology. 1987;37: 1837-44.

2. Scher MS, Aso K, Beggarly ME, Hamid MY. Electrographic seizures in preterm and full term neonates: Clinical correlates, associated brain lesions, and risk for neurologic sequelae. Pediatrics.1993;91:128-34.

3. Biagioni E, Ferrari F, Boldrini A, Roversi MF, Cioni G. Electroclinical correlation in neonatal seizures. Eur $\mathrm{J}$ Peadiatr Neurol. 1998;2:117-25. 